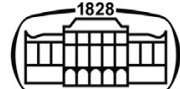

AKADÉMIAI KIADÓ

Journal of Behavioral Addictions

9 (2020) 1, 71-82

DOI:

$10.1556 / 2006.2020 .00003$

(c) 2020 The Author(s)

\section{FULL-LENGTH REPORT}

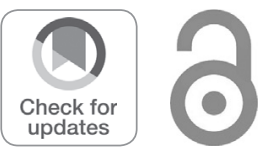

\title{
Response inhibition during processing of sexual stimuli in males with problematic hypersexual behavior
}

JI-WOO SEOK ${ }^{1,2}$, and JIN-HUN SOHN ${ }^{1 *}$ ๑

\author{
${ }^{1}$ Department of Psychology, Chungnam National University, Daejeon, 305-764, South Korea \\ ${ }^{2}$ Department of Rehabilitation Counseling Psychology, Seoul Hanyoung University, Seoul, 08274, \\ South Korea
}

Received: July 14, 2019 • Revised manuscript received: October 6, 2019; December 13, 2019 • Accepted: December 21, 2019 - Published online: April 7, 2020

\begin{abstract}
Background and aims: Individuals with problematic hypersexual behavior (PHB) are unable to control their sexual cravings, regardless of other situational factors. This inability to control cravings is a common trait in patients with neurological pathologies related to response inhibition. Until recently, however, it was unclear whether individuals with PHB have decreased inhibition and altered neural responses in the brain regions associated with inhibition compared to healthy control individuals, especially in the presence of distracting sexual stimuli. In this study, we examined the neural and psychological underpinnings of inhibition in individuals with PHB. Methods: Thirty individuals with PHB and 30 healthy subjects underwent functional magnetic resonance imaging while performing a modified go/no-go task with neutral or sexual backgrounds used as distractors. Results: Individuals with PHB showed poorer response inhibition than healthy subjects, especially when sexual distractors were present. Further, compared to healthy control subjects, individuals with PHB showed decreased activation in the right inferior frontal gyrus (IFG) and reduced functional connectivity between the IFG and the pre-supplementary motor area (preSMA) when response inhibition was required. Finally, the reduced activation and connectivity were more pronounced in the presence of sexual distractors than in the presence of neutral distractors. Discussion: These findings suggest that individuals with PHB show reduced ability to inhibit responses that might be related to lower IFG activation and IFG-preSMA connectivity during response inhibition. Our results provide insights into the neurobiological underpinnings of poor response inhibition in individuals with PHB.
\end{abstract}

\section{KEYWORDS}

problematic hypersexual behavior, response inhibition, functional magnetic resonance imaging, functional connectivity, inferior frontal gyrus

\section{INTRODUCTION}

Individuals with problematic hypersexual behavior (PHB) have been reported to demonstrate high impulsivity, cognitive rigidity, poor judgment, deficits in emotion regulation, and an excessive preoccupation with sex (Carnes, 2001; Kafka, 2010). Individuals with PHB are unable to control their sexual cravings, regardless of other situational factors (Carnes, 2001; Kafka, 2010). These behavioral characteristics indicate that individuals with PHB might have problems with inhibition, but little research has been conducted on this issue. The current study attempted to address that knowledge gap.

Inhibition of interfering information is essential in order to focus attention, eliminate inappropriate memories to retrieve relevant memories, and suppress habitual responses to make adaptive choices. Inhibition is a prerequisite for obtaining the desired information in a
${ }^{*}$ Corresponding author. Tel.: +8242 821 7404; fax: +82 428218875 E-mail: jhsohn@cnu.ac.kr 
changing environment and impaired inhibitory control is associated with severe negative consequences (Dillon \& Pizzagalli, 2007).

Neuroimaging studies using stop signal tasks and go/nogo tasks have shown that brain regions associated with successful response inhibition include the inferior frontal gyrus (IFG), insula, pre-supplementary motor area (preSMA), dorsal anterior cingulate cortex (ACC), parietal lobe, and subthalamic nucleus (Aron, Monsell, Sahakian, \& Robbins, 2004; Duann, Ide, Luo, \& Li, 2009; Simmonds, Pekar, \& Mostofsky, 2008; Swick, Ashley, \& Turken, 2011). The right IFG in particular plays a pivotal role in inhibitory control, showing higher percent signal changes during tasks that requires response inhibition than during automatic responses (Aron, Monsell, Sahakian, \& Robbins, 2004; Menon, Adleman, White, Glover, \& Reiss, 2001; Rubia et al., 2001). Furthermore, findings from brain lesion and transcranial magnetic stimulation studies indicate that the ability to control an automatic response is associated with the integrity of the right IFG and the preSMA (Aron, Behrens, \& Smith, 2007; Chikazoe, 2010; Levy \& Wagner, 2011; Nachev, Kennard, \& Husain, 2008). Diffusion tensor imaging (DTI) tractography has revealed that the IFG and the preSMA are anatomically connected to one another and that, together with the basal ganglia, they constitute a network for action control (Aron, Behrens, \& Smith, 2007; Ford, McGregor, Case, Crosson, \& White, 2010; Forstmann et al., 2010). Functional magnetic resonance imaging (fMRI) studies have shown that both areas are activated during the preparation for stopping as well as during outright stopping (Chikazoe et al., 2009; Jahfari, Stinear, Claffey, Verbruggen, \& Aron, 2010; Zandbelt \& Vink, 2010). This may indicate that one or both regions are involved in preparing the "stopping network" in advance so that inhibitory control can subsequently be triggered during the detection of the stop signal (Swann et al., 2012). These results provide convergent evidence supporting the view that decreased inhibitory control on nogo trials is associated with the hypoactivation of the right IFG and the preSMA and altered functional connectivity between the two.

Previous studies have revealed correlations between diminished control and other addictive behaviors such as internet gaming disorder (Ding et al., 2014; Dong, Devito, Du, \& Cui, 2012; Liu et al., 2014) and gambling disorder (Odlaug, Chamberlain, Kim, Schreiber, \& Grant, 2011; Van Holst, Van Holstein, Van Den Brink, Veltman, \& Goudriaan, 2012). In these studies, participants with behavioral addictions showed decreased response inhibition compared to healthy controls, and the impairment was related to altered activation in the prefrontal cortex, anterior cingulate cortex, and parietal lobe. Additionally, participants with behavioral addictions showed much more diminished response inhibition during the performance of an inhibition task in the context of an addiction-related stimulus (Liu et al., 2014; Van Holst, Van Holstein, Van Den Brink, Veltman, \& Goudriaan, 2012). The authors thus suggested that an addiction-related context is more salient for participants with behavioral addictions and affects their response inhibition, as indicated by altered brain activity and behavioral error patterns.

Several studies investigated inhibition abilities in individuals with PHB (Miner, Raymond, Mueller, Lloyd, \& Lim, 2009; Reid, Garos, \& Carpenter, 2011a; Reid, Garos, Carpenter, \& Coleman, 2011b; Reid, Karim, McCrory, \& Carpenter, 2010; Seok \& Sohn, 2018). Reid, Karim, McCrory, \& Carpenter, 2010 identified self-reported executive dysfunctions including response inhibition among hypersexual patients, while, in another study using neuropsychological tests, they (2011) did not find general impairments of executive functions. However, several other studies reported reduced inhibition in individuals with $\mathrm{PHB}$ compared to healthy controls (Miner, Raymond, Mueller, Lloyd, \& Lim, 2009; Seok \& Sohn, 2018). Miner, Raymond, Mueller, Lloyd, \& Lim, 2009 used DTI to show decreased inhibition control during go/no-go tasks and significantly higher superior frontal region mean diffusivity in individuals with PHB compared to control subjects. Another study combining the Stroop task and fMRI observed diminished executive control in participants with $\mathrm{PHB}$ and identified the underlying neural mechanisms (Seok \& Sohn, 2018). In all these studies, the authors investigated general response inhibition in individuals with $\mathrm{PHB}$ using affective neutral task paradigms.

However, cue-reactivity and executive functioning, including response inhibition, have been shown to influence each other in a synergistic way in patients with addiction (Dawe, Gullo, \& Loxton, 2004; Luijten, Littel, \& Franken, 2011). This indicates that individuals with stronger cue reactivity may have more problems with inhibition behavior. Over the course of their addiction, patients develop an attraction towards addiction-related stimuli as they tend to catch their attention (Field \& Cox, 2008; Franken, 2003). Previous studies of addiction (to drugs and alcohol) suggest that an abnormal increase in the attention and motivation related to salient objects could transform the addiction into a disinhibitory disorder and lead to loss of control over substance use and relapse (Cox, Hogan, Kristian, \& Race, 2002; Noël et al., 2007). In line with these considerations, the finding of an attentional bias toward the salient stimuli representing the addiction (in this case, sexually explicit cues) among participants with $\mathrm{PHB}$ has been replicated (Mechelmans et al., 2014), suggesting that executive functions including response inhibition in individuals with $\mathrm{PHB}$ may be affected in situations when they are confronted with the sexually explicit cues eliciting craving reactions. However, relatively little is known about the relationship between cue-reactivity and response inhibition and the neural underpinning of this relationship in PHB.

Therefore, we tested whether individuals with PHB show particularly poor inhibitory control in the presence of sexrelated cues, using the modified go/no-go task developed by Telzer, Fuligni, Lieberman, \& Galván, 2013. We hypothesized that individuals with $\mathrm{PHB}$ would show reduced response inhibition compared to controls and, specifically, that inhibitory control might be more reduced during direct exposure to sex-related stimuli in individuals with PHB. We 
also investigated the neural mechanisms underlying diminished inhibitory control among individuals with PHB, hypothesizing that they would show altered activation in the right IFG and preSMA compared to healthy controls.

\section{METHODS}

\subsection{Participants}

All participants were recruited via advertising posts on internet bulletin boards or at treatment centers for problematic sexual behavior and during Sex Addicts Anonymous meetings. All participants were native Korean speakers, male, and right-handed, and had no history of illegal substance use. We chose participants based on specific inclusion and exclusion criteria (see more details in the Supplementary materials). Individuals with PHB were diagnosed by a psychiatrist using a structured interview detailed in Table S1, as in previous studies (Carnes, Green, \& Carnes, 2010; Kafka, 2010). To assess the degree of PHB of the participants, standardized questionnaires such as the Sexual Addiction Screening Test-R (SAST-R) (Carnes et al., 2010) and the Hypersexual Behavior Inventory (HBI) (Reid et al., 2011a) were used (see Supplementary materials). Furthermore, standardized inventories such as the Barratt Impulsiveness Scale-11 (BIS) (Patton, Stanford, \& Barratt, 1995), and the Beck Depression Inventory (BDI) (Beck, Steer, \& Brown, 1996) were used to evaluate the psychological characteristics of the participants.

To delineate the effect of hypersexuality on response inhibition and rule out any factors that could affect neural and cognitive responses, we selected several exclusion criteria based on previous studies (Anand et al., 2005; Chen, Tien, Juan, Tzeng, \& Hung, 2005; Hester \& Garavan, 2004; Kaufman, Ross, Stein, \& Garavan, 2003; Le Marquand et al., 1998) (see Supplementary materials). Thirty men with PHB (mean age $=28.81$ years, standard deviation $[\mathrm{SD}]=5.26$ years) were recruited after applying these criteria.
For the control group, 30 participants (mean age $=27.41$ years, $\mathrm{SD}=4.01$ years) with matching demographic characteristics (age, sex, education level, and income level) were selected.

All participants provided written informed consent after being provided an explanation about the study. The experimental and consent procedures were approved by the Chungnam National University Institutional Review Board (approval number: 201309-SB-003-01). All participants received financial compensation $(100,000 \mathrm{KRW})$ for their participation in the study.

All participants completed a survey containing questions related to demographics and sexual behavior. The questions concerning sexual behavior included age at first sexual intercourse, average weekly frequency of sexual intercourse, average weekly masturbation frequency, weekly frequency of viewing pornography, and total number of sex partners over the previous six months.

\subsection{Experimental Stimuli and Paradigm}

To study response inhibition in the present study, we modified the go/no-go task developed by Telzer, Fuligni, Lieberman, \& Galván, 2013 and implemented it using fMRI and E-prime software (Psychology Software Tools, Inc., Pittsburgh, PA). All participants were presented with the same behavioral paradigm while they were undergoing an fMRI scan. Participants performed 360 trials (135 go trials with neutral background, 135 go trials with sexual background, 45 no-go trials with neutral background, and 45 nogo trials with sexual background) over the course of nine blocks during one functional run. Each block lasted $70 \mathrm{~s}$, with an interval of $12 \mathrm{~s}$ between blocks. Each block included 10 no-go trials and 30 Go trials. The background images (sexual and neutral) were presented in random order. Each trial was presented for $1000 \mathrm{~ms}$, with an intertrial interval jittered according to a random gamma distribution (average $=750 \mathrm{~ms}$ ).

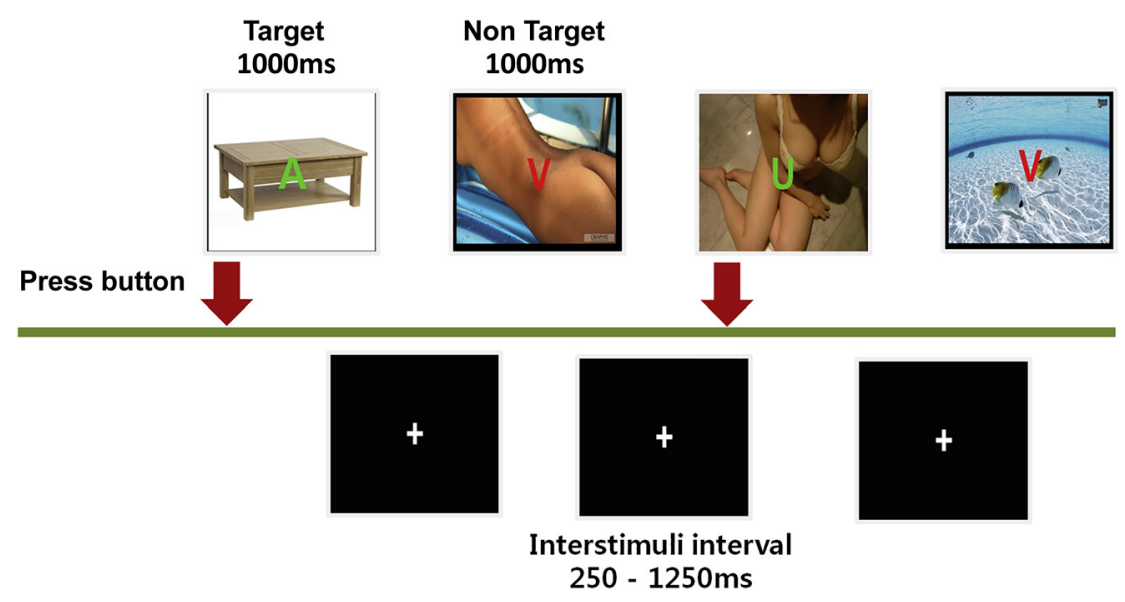

Figure 1. Experimental design. Example of trials in the modified go/no-go task 
Participants were presented with a series of rapid trials, each displaying a single letter, and were instructed to press a button in response to all letters except for "V" as quickly as possible. The letter $\mathrm{V}$ was presented in $25 \%$ of the trials. Unlike Telzer, Fuligni, Lieberman, \& Galván, 2013, the present experiment used sexual and neutral photos as background images, with target and non-target stimuli presented in the center of a sexual or neutral image instead of a black screen. Thus, participants developed a pre-potent response to press a button when the target stimulus was presented (go trials) and had to suppress the go response when non-target trials were presented (no-go trials), regardless of the background (i.e., sexual or neutral). We conducted a pilot study to validate the sexual and neutral photos (see Supplementary materials). Examples of the modified go/no-go stimuli and the stimulus-presenting paradigm are shown in Fig. 1 and in the Supplementary materials.

\subsection{Image Acquisition}

Participants saw the presented stimuli on an overhead mirror while undergoing functional MRI. An echo-planar imaging blood oxygen level-dependent (EPI-BOLD) method was used to acquire brain images on a $3.0 \mathrm{~T}$ Philips MR scanner. The parameters for image acquisition were as follows: repetition time $(\mathrm{TR})=2000 \mathrm{~ms}$; echo time $(\mathrm{TE})=28 \mathrm{~ms}$, field of view $($ FOV $)=240 \times 240 \mathrm{~mm}^{2} ;$ matrix $=64 \times 64$; slice thickness $=5 \mathrm{~mm}$ with no gap; and flip angle $=80^{\circ}$. A total of 369 images, including three dummy images, were acquired during the experiment. T1-weighted structural images were also collected $\left(\mathrm{TR} / \mathrm{TE}=280 / 14 \mathrm{~ms}, \mathrm{FOV}=240 \times 240 \mathrm{~mm}^{2}\right.$; matrix $=256 \times 256$; slice thickness $=4 \mathrm{~mm}$; flip angle $=60^{\circ}$ ).

\subsection{Statistical Analysis}

2.4.1. Task performance. Three-way analysis of variance (ANOVA) was performed to test influences of (1) the within-subjects variables, namely background (sexual and neutral) and trial (go and no-go); and (2) the betweensubjects variable group (PHB and control) on each of the dependent variables, namely total error rate and response time on the go/no-go task. All statistical analyses were performed using SPSS 25.0.

2.4.2. Task-based imaging. Imaging data were preprocessed and analyzed using the Statistical Parametric Mapping software (SPM12, http://www.fil.ion.ucl.ac.uk/spm). The first five volumes were discarded. Functional data were realigned to the first scan of each session as reference. Head motion above 2 $\mathrm{mm}$ in any direction was an exclusion criterion, but no participant was excluded on this basis. The realigned scans were co-registered to individual anatomical images and normalized onto the Montreal Neurologic Institute template brain. During normalization, images were resampled every 4 $\mathrm{mm}$ using sinc interpolation and smoothed with an 8-mm isotropic Gaussian kernel to decrease spatial noise.

Statistical analyses were performed on a voxel-wise basis across the whole brain using standard methodology in SPM12.
Individual events were modeled by a synthetic hemodynamic response function (HRF). Four regressors were used to model the experimental conditions: go trials on a sexual background; go trials on a neutral background; no-go trials on a sexual background; and no-go trials on a neutral background. To account for residual movement artifacts after realignment, movement parameters derived from realignment corrections were entered as covariates of no interest. Statistical parametric maps were generated from linear contrasts between the HRF parameter estimates for the different types of trials (i.e., go or no-go) and backgrounds (i.e., sexual or neutral background).

Three-way ANOVAs were conducted with group, type of trial, and background as the independent variables in order to examine main effects and interaction effects among these variables on brain activation. Effects were considered statistically significant at an FDR-corrected height threshold of $P<0.05$ and an extent threshold of 20 voxels. To confirm the validity of the results acquired through SPM, follow-up $F$-tests were conducted using percent signal change values in regions showing significant interaction effects.

We then performed a two-way ANOVA on the brain activation for each trial type, to find a background $\times$ group interaction effect (Supplementary materials, Tables S2 and S3). Moreover, a correlation analysis was conducted using data from individuals with $\mathrm{PHB}$ to identify the correlation between the IFG during no-go trials with sexual backgrounds and the severity of hypersexual behavior as measured by the HBI score (see Supplementary materials).

2.4.3. Functional connectivity. Functional connectivity between two regions of interest (ROIs), namely the right IFG and preSMA, was calculated, as these regions are known to be associated with response inhibition (Chikazoe et al., 2009; Jahfari, Stinear, Claffey, Verbruggen, \& Aron, 2010; Swann et al., 2012; Zandbelt \& Vink, 2010). The mask images of both ROIs (right IFG and preSMA) were defined using the WFU PickAtlas tool (Wake Forest University, Wake Forest, NC). Signals from all voxels within these masks were extracted and averaged, and the correlation between the averaged signals for the two ROIs was assessed with Pearson's correlation coefficient in each condition (i.e., sexual no-go condition, sexual go condition, neutral no-go condition, and neutral go condition).

Three-way ANOVA was conducted with group, type of trial, and background as the independent variables in order to examine main effects and interaction effects among these variables on the IFG-preSMA connectivity.

In addition, a separate correlation analysis was conducted using data from individuals with PHB to identify the link between IFG-preSMA connectivity during no-go trials with sexual backgrounds and the severity of hypersexual behavior as measured by the HBI score (see Supplementary materials).

\subsection{Ethics}

All study procedures were conducted in accordance with the ethical guidelines of institutional review board and the Declaration of Helsinki. 
Table 1. Description of demographic data, variables concerning sexual behavior, and scores on standardized scales among PHB and control groups

\begin{tabular}{|c|c|c|c|}
\hline & Control $(N=30)$ & PHB $(N=30)$ & $t$ or chi square \\
\hline Age (years) & $27.41(4.01)$ & $28.81(5.26)$ & 1.15 \\
\hline Years of education & $15.07(2.50)$ & $15(2.61)$ & 0.11 \\
\hline \multicolumn{4}{|l|}{ Marital status ${ }^{1}$} \\
\hline Single & 66.67 & 56.67 & 0.680 \\
\hline In a relationship & 23.33 & 33.33 & \\
\hline Engaged/married & 10 & 10 & \\
\hline Number of sexual partners ${ }^{2}$ & $2.67(2.15)$ & $14.8(12.45)$ & $5.29^{* * *}$ \\
\hline Weekly frequency of sexual intercourse ${ }^{2}$ & $1.90(1.54)$ & $4.80(1.35)$ & $7.57^{* * *}$ \\
\hline Weekly frequency of masturbation ${ }^{2}$ & $1.77(1.22)$ & $4.43(1.55)$ & $7.28^{* * *}$ \\
\hline $\begin{array}{l}\text { Weekly frequency of viewing } \\
\text { pornography }\end{array}$ & $1.80(1.19)$ & $5.23(1.17)$ & $11.32^{* * *}$ \\
\hline $\begin{array}{l}\text { Sexual Addiction Screening } \\
\text { Test-R scores }\end{array}$ & $0.57(0.62)$ & $12.90(3.49)$ & $19.05^{\star * *}$ \\
\hline Hypersexual Behavior Inventory scores ${ }^{2}$ & $24.17(5.62)$ & $61.07(8.40)$ & $19.99^{* * *}$ \\
\hline Beck Depression Inventory scores ${ }^{2}$ & $9.08(6.55)$ & $4.01(3.82)$ & $3.66^{* *}$ \\
\hline Barrett's Impulsiveness Scale scores ${ }^{2}$ & $54.03(6.82)$ & $46.10(5.01)$ & $5.13^{* * *}$ \\
\hline
\end{tabular}

SD: Standard deviation.

${ }^{\star} P<0.05,{ }^{* *} P<0.01,{ }^{* *} P<0.001$.

${ }^{1}$ Data are represented as percentages.

${ }^{2}$ Data are represented as means (SD).

\section{RESULTS}

\subsection{Demographic Characteristics and Task Performance}

The demographic characteristics and background information of the participants are shown in Table 1.

Behavioral performance was assessed by calculating error rates as an index of impaired response inhibition. A three-way ANOVA with trial (go vs. no-go) and background (neutral vs. sexual) as within-subject factors and group (controls vs. PHB group) as between-subject factor yielded significant main effects of background $(F(1,232)=37.70, P<0.001)$ and trial $(F(1$, $232)=124.00, P<0.001)$ (Fig. 2). Most importantly, differences between the groups were observed $(F(1,232)=84.30, P<$ 0.001). A main effect of group exhibited that individuals with PHB (mean $=0.12$ ) performed worse than control subjects $($ mean $=0.04)$. We also found significant interaction effects of group $\times$ background $\times$ trial $(F(1,232)=4.21, P<0.05)$, group $\times$ background $(F(1,232)=17.19, \quad P<0.001)$, group $\times$ trial
$(F(1,232)=29.84, P<0.001)$ and background $\times$ trial $(F(1$, $232)=12.21, P<0.01)$. Figure 2 shows the significant interaction effects of group $\times$ background on no-go trials $(F(1,116)=$ $12.83, P<0.001)$ and on go trials $(F(1,116)=4.36, P<0.05)$.

Further, we performed a two-way ANOVA on response times and found a significant background $\times$ group interaction effect on response times in the go trials $(F(1,116)=$ 6.49, $P<0.05$; Fig. S1).

\subsection{Task-based Imaging}

3.2.1. Main effect of background. We found a significant main effect of background (sexual vs. neutral) in the fusiform gyrus, middle occipital gyrus, medial frontal gyrus, IFG, insula, cuneus, and inferior parietal lobule $(P<0.05$, FDR-corrected; Table 2). These regions showed greater activation when seen on sexual backgrounds than when seen on neutral backgrounds. No brain regions showed higher activations in response to neutral backgrounds than sexual backgrounds.
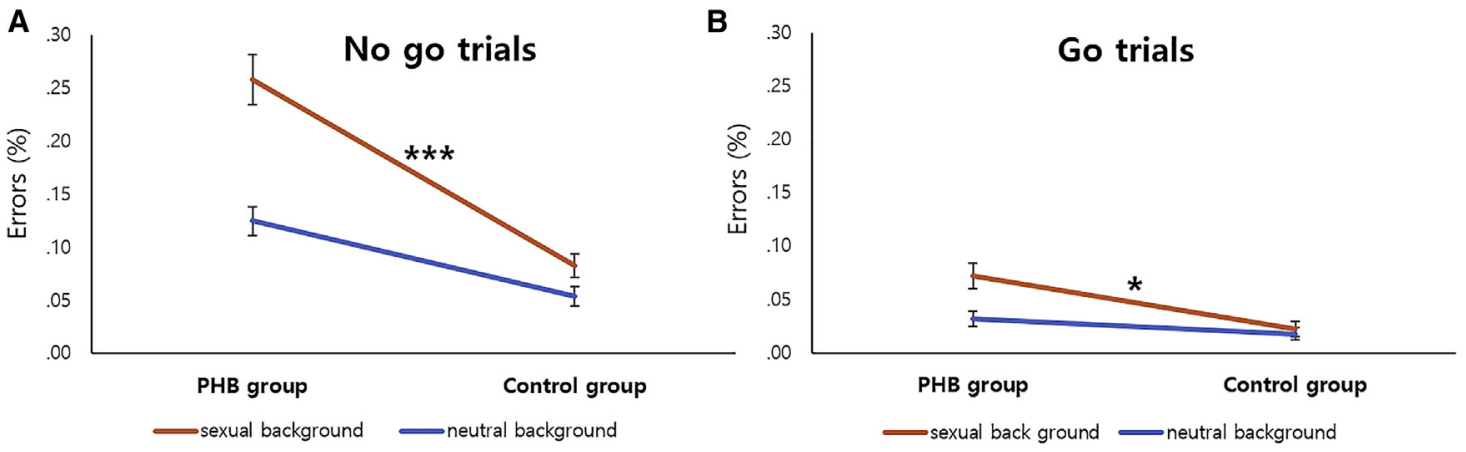

Figure 2. Behavioral results. (A) Error ratio on no-go trials. (B) Error ratio on go trials. Red and blue indicate the values for the sexual background and the neutral background, respectively. Error bars show the standard errors of the means. ${ }^{\star} P<0.05,{ }^{* *} P<0.001$ 
Table 2. Main effects of background, trial type, and group

\begin{tabular}{|c|c|c|c|c|c|c|c|}
\hline \multicolumn{3}{|c|}{ Coordinates } & \multirow[b]{2}{*}{ Side } & \multirow[b]{2}{*}{ Brain regions } & \multirow[b]{2}{*}{$\mathrm{BA}$} & \multirow[b]{2}{*}{ Cluster size } & \multirow[b]{2}{*}{$F$} \\
\hline$x$ & $y$ & $z$ & & & & & \\
\hline \multicolumn{8}{|c|}{$\begin{array}{l}\text { Main effect of background } \\
\text { Sexual }>\text { Neutral }\end{array}$} \\
\hline-40 & -62 & -10 & $\mathrm{~L}$ & Fusiform gyrus & 37 & 2015 & 38.25 \\
\hline 43 & -65 & 7 & $\mathrm{R}$ & Middle occipital gyrus & 19 & 1540 & 23.76 \\
\hline 50 & 9 & 26 & $\mathrm{R}$ & Inferior frontal gyrus & 44 & 380 & 18.93 \\
\hline 44 & 14 & 11 & $\mathrm{R}$ & & & 224 & 17.66 \\
\hline 5 & -72 & 21 & $\mathrm{R}$ & Cuneus & 17,18 & 210 & 17.43 \\
\hline-11 & -69 & 16 & $\mathrm{~L}$ & & & 148 & 15.82 \\
\hline 30 & 34 & 26 & $\mathrm{R}$ & Medial frontal gyrus & 9 & 160 & 16.12 \\
\hline 38 & 10 & 1 & $\mathrm{R}$ & Insula & 13 & 79 & 15.11 \\
\hline 45 & -51 & 40 & $\mathrm{R}$ & Inferior parietal lobule & -39 & 70 & 14.68 \\
\hline \multicolumn{8}{|c|}{$\begin{array}{l}\text { Sexual }<\text { Neutral } \\
\text { No regions } \\
\text { Main effect of trial type } \\
\text { Go }>\text { No-go }\end{array}$} \\
\hline 43 & -27 & 45 & $\mathrm{R}$ & Postcentral gyrus & 1,2 & 1456 & 20.60 \\
\hline 45 & -11 & 55 & $\mathrm{R}$ & Precentral gyrus & 4 & 144 & 17.51 \\
\hline 46 & 8 & -0 & $\mathrm{R}$ & Insula & 13 & 218 & 15.49 \\
\hline $\begin{array}{c}-42 \\
\mathrm{Go}<\mathrm{No}\end{array}$ & 7 & 1 & $\mathrm{~L}$ & & & 169 & 14.27 \\
\hline 43 & 28 & 12 & $\mathrm{R}$ & Inferior frontal gyrus & 46 & 49 & 12.56 \\
\hline \multicolumn{8}{|c|}{ Main effect of group } \\
\hline 17 & 0 & 2 & $\mathrm{R}$ & Globus pallidus & - & 1393 & 33.67 \\
\hline 39 & -7 & -4 & $\mathrm{R}$ & & & 352 & 25.31 \\
\hline-15 & 8 & 4 & $\mathrm{~L}$ & Claustrum & & 709 & 28.29 \\
\hline 9 & 2 & 32 & $\mathrm{R}$ & Cingulate gyrus & $23,24,31$ & 6396 & 32.19 \\
\hline 6 & -45 & 23 & $\mathrm{R}$ & & & 1569 & 30.45 \\
\hline-12 & -55 & 38 & $\mathrm{~L}$ & Posterior cingulate gyrus & & 651 & 26.13 \\
\hline 42 & -10 & 36 & $\mathrm{R}$ & Precentral gyrus & $1,4,6$ & 215 & 19.85 \\
\hline-44 & -25 & 40 & $\mathrm{~L}$ & Postcentral gyrus & & 420 & 26.53 \\
\hline 38 & -52 & 23 & $\mathrm{R}$ & Middle temporal gyrus & 39 & 189 & 17.48 \\
\hline 44 & -52 & -9 & $\mathrm{R}$ & Fusiform gyrus & 37 & 602 & 17.30 \\
\hline \multicolumn{8}{|c|}{ PHB $<$ Control } \\
\hline 28 & -10 & 52 & $\mathrm{R}$ & Supplementary motor area & 6 & 78 & 29.67 \\
\hline 39 & -20 & -18 & $\mathrm{R}$ & Inferior frontal gyrus & 47 & 52 & 11.92 \\
\hline-52 & -38 & 24 & $\mathrm{~L}$ & Inferior parietal lobule & 40 & 42 & 11.06 \\
\hline
\end{tabular}

$\mathrm{BA}=$ Brodmann area; $\mathrm{L}=$ left hemisphere; $\mathrm{R}=$ right hemisphere; $\mathrm{PHB}=$ problematic hypersexual behavior; and $\mathrm{FDR}=$ false discovery rate. $P<0.05$ (FDR-corrected).

3.2.2. Main effect of trial type. We found a significant main effect of trial type (go vs. no-go) in the precentral gyrus, postcentral gyrus, and insula. These regions showed greater activation during go trials than no-go trials. In addition, the right IFG showed higher activation during nogo trials than go trials $(P<0.05$, FDR-corrected; Table 2$)$.

3.2.3. Main effect of group. We observed a significant main effect of group (individuals with $\mathrm{PHB}$ vs. control subjects; $P$ $<0.05$, FDR-corrected; Table 2) across the brain, including regions such as the striatum (globus pallidus and claustrum), cingulate gyrus (anterior and posterior), precentral gyrus, postcentral gyrus, middle temporal gyrus, and fusiform gyrus. In contrast, healthy controls showed higher activation in the preSMA, IFG, and inferior parietal lobule compared to the PHB group.

3.2.4. Interaction effects. The three-way ANOVA yielded a significant background $\times$ trial type interaction in the right precuneus (Brodmann's area 7), IFG, insula, and preSMA (Brodmann's area 6) $(P<0.05$, FDR-corrected; Table 3). We also identified a significant interaction effect of background $\times$ group in the right IFG and fusiform gyrus, and a 
Table 3. Interaction effects of background, trial type, and group

\begin{tabular}{|c|c|c|c|c|c|c|c|}
\hline \multicolumn{8}{|c|}{ Coordinates } \\
\hline$x$ & $y$ & $z$ & Side & Brain regions & BA & Cluster & $F$ \\
\hline \multicolumn{8}{|c|}{ Interaction effect of background $\times$ trial type } \\
\hline 23 & -50 & 61 & $\mathrm{R}$ & Precuneus & 7 & 77 & 17.42 \\
\hline 20 & 7 & 51 & $\mathrm{R}$ & $\begin{array}{l}\text { Presupplementary } \\
\text { motor area }\end{array}$ & 6 & 71 & 17.37 \\
\hline 40 & 20 & -16 & $\mathrm{R}$ & Inferior frontal gyrus & 47 & 68 & 16.11 \\
\hline 40 & 12 & 6 & $\mathrm{R}$ & Insula & 13 & 63 & 14.98 \\
\hline \multicolumn{8}{|c|}{ Interaction effect of group $\times$ trial type } \\
\hline 45 & 30 & -16 & $\mathrm{R}$ & Inferior frontal gyrus & 46 & 59 & 14.84 \\
\hline \multicolumn{8}{|c|}{ Interaction effect of group $\times$ background type } \\
\hline 38 & 22 & -12 & $\mathrm{R}$ & Inferior frontal gyrus & 47 & 63 & 15.07 \\
\hline 40 & -57 & -9 & $\mathrm{R}$ & Fusiform gyrus & 37 & 60 & 14.56 \\
\hline \multicolumn{8}{|c|}{ Interaction effect of background $\times$ trial type $\times$ group } \\
\hline 42 & 32 & -12 & $\mathrm{R}$ & Inferior frontal gyrus & 47 & 112 & 17.13 \\
\hline
\end{tabular}

$\mathrm{BA}=$ Brodmann area; and $\mathrm{FDR}=$ false discovery rate.

$P<0.05$, FDR-corrected.

significant interaction effect of group $\times$ trial type in the right IFG $(P<0.05$, FDR-corrected; Table 3$)$.

Further, we found a significant three-way group $\times$ trial type $\times$ background interaction effect $(P<0.05$, FDRcorrected; Table 3, Fig. 3) in the IFG. The follow-up F-tests using the percent signal change data of the IFG revealed significant group $\times$ background $\times$ trial $(F(1,232)=4.82$, $P<0.05)$, group $\times$ background $(F(1,232)=4.94, P<0.05)$, group $\times$ trial $(F(1,232)=6.13, P<0.05)$, and background $\times$ trial $(F(1,232)=14.44, P<0.001)$ interaction effects on the IFG activation. We also found significant main effects of background $(F(1,232)=11.0, P<0.001)$, group $(F(1,232)=30.65, P<0.001)$, and trial $(F(1,232)=315.50$, $P<0.001)$ on the IFG activation. Figure 3 shows the average percent signal changes in the IFG for each condition and the observed within-group and between-group differences.
A
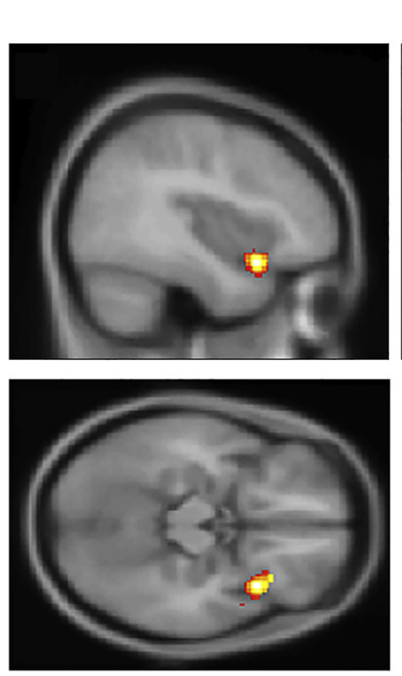

B

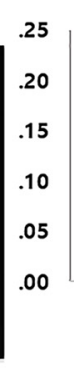

C

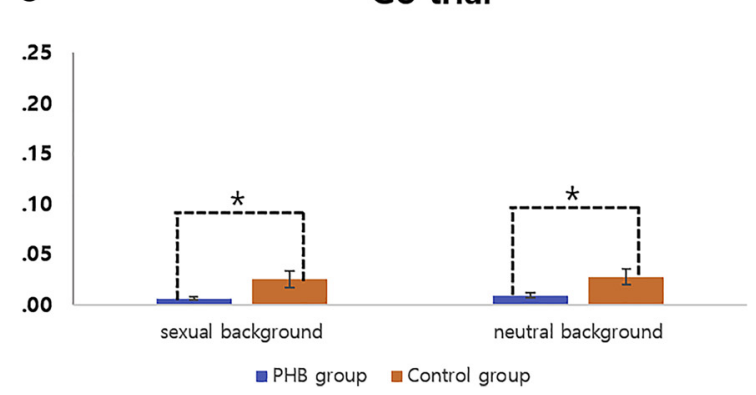

Figure 3. Brain areas showing the background $\times$ trial type $\times$ group interaction effect during go/no-go task performance $(P<0.05$, FDRcorrected). (A) Inferior frontal gyrus $(x=42, y=32, z=-12)$. (B) Average percent signal change for each background on go trials. (c) Average percent signal change for each background on no-go trials. Red and blue indicate the means of values for the control group and PHB group, respectively. Error bars show the standard errors of the means. ${ }^{\star} P<0.05,{ }^{\star *} P<0.001$ 
A

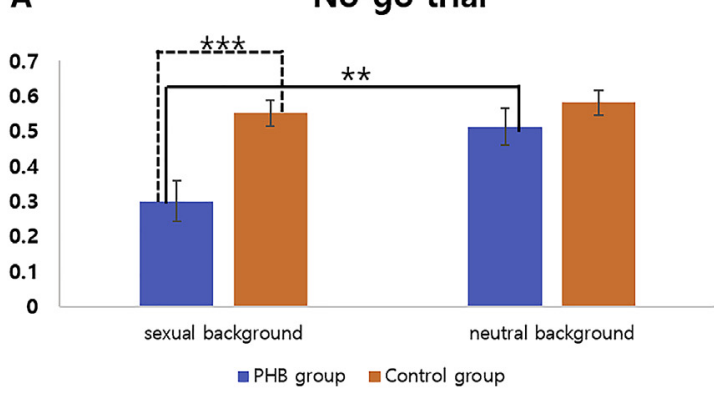

B

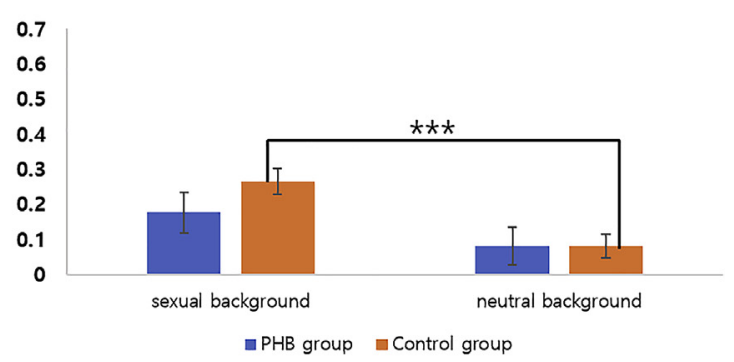

Figure 4. The background $\times$ group interaction effect on IFG-preSMA connectivity for each trial type. (A) Average IFG-preSMA correlation coefficient for no-go trials. (B) Average IFG-preSMA correlation coefficient for go trials. Red and blue indicate the means of values for the neutral background and the sexual background, respectively. Error bars show the standard errors of the means. ${ }^{\star} P<0.05$

\subsection{Functional Connectivity}

3.3.1. IFG-preSMA connectivity in each condition. We found no significant functional connectivity between the IFG and preSMA during the go task in the control group (correlation coefficients: neutral background $=0.08$; sexual background $=0.27 ; P>0.05$ ) or in the PHB group (correlation coefficients: neutral background $=0.08$; sexual background $=0.18 ; P>0.05$ )

When control subjects controlled their predominant reaction (i.e., during the no-go trials), they showed significant positive functional connectivity between the right IFG and the right preSMA, irrespective of background (correlation coefficients: neutral background $=0.58$; sexual background $=$ $0.54 ; P<0.01)$, suggesting that strong connectivity between the IFG and preSMA was associated with successful response inhibition. In contrast, individuals with $\mathrm{PHB}$ showed positive functional connectivity between the IFG and preSMA only when they performed no-go trials with a neutral background (correlation coefficient $=0.51 ; P<0.05$ ); in other words, as participants with PHB made many errors while being presented with the sexual backgrounds, there was no significant connectivity between the two regions (correlation coefficient $=0.32 ; P>0.05$ ).

\subsubsection{Three-way ANOVA results on IFG-preSMA connec-} tivity. We found a significant main effect of group $(F(1,232)=9.73, P<0.01)$ and trial $(F(1,232)=104.13, P$ $<0.001)$ on the IFG-preSMA connectivity. Further, we found a significant group $\times$ trial type $(F(1,232)=4.26$, $P<0.05)$ interaction effect and a group $\times$ background interaction effect $(F(1,232)=15.73, P<0.001)$ on the IFGpreSMA connectivity. Figure 4 shows the average IFG-preSMA correlation coefficients for each condition and the observed within-group and between-group differences.

\section{DISCUSSION}

The present study used fMRI to investigate the neural substrates of inhibition during go/no-go tasks presented on neutral or sexually charged backgrounds in individuals with PHB. Our behavioral results show generally poor response inhibition in individuals with $\mathrm{PHB}$, that was predominantly observed on trials with sexual backgrounds. The brain imaging results show reduced brain activation in the right IFG in individuals with $\mathrm{PHB}$ when response inhibition was required, and this dysfunction in the IFG was more predominant when sexual backgrounds were presented. Also, our results suggest that individuals with $\mathrm{PHB}$ show decreased functional connectivity between the right IFG and the right preSMA in response to sexual backgrounds.

The PHB group made more errors during the task than the control group, regardless of the background. This suggests that individuals with $\mathrm{PHB}$ may be unable to inhibit automatic responses (Aron, Behrens, Smith, Frank, \& Poldrack, 2007). Response inhibition refers to the ability to inhibit explicit behaviors usually involving impulsive responses. It is also the ability to smoothly transform responsive behaviors into other behaviors in response to environmental changes, which is necessary to suppress automatic/dominant responses and maintain goal-oriented behavior (Harnishfeger, 1995). The difficulty individuals with $\mathrm{PHB}$ have inhibiting a dominant response on the go/ no-go task used in our study thus supports the results of previous studies (Miner, Raymond, Mueller, Lloyd, \& Lim, 2009; Seok \& Sohn, 2018).

The observed interference with response inhibition might be related to psychological characteristics such as impulsivity (Antons \& Brand, 2018; Volkow et al., 2013). Volkow suggested that addiction-related stimuli might increase the activity of the impulsive system and lead to impulsive action tendencies. This suggestion was supported by the findings of Antons and Brand (2018). The authors investigated the relationships between symptom severity of Internet pornography-use disorder and trait and state impulsivity measured with the short version of the German Barratt Impulsiveness Scale (BIS-15; Meule, Vögele, \& Kübler, 2011) and a modified stop-signal task, respectively. They found that trait impulsivity was linked with higher symptom severity of Internet pornography-use disorder, and trait impulsivity interacts with state impulsivity, resulting in an accumulating effect on symptom severity (Antons \& Brand, 2018).

In line with this finding, we found that the PHB group had higher trait impulsivity, as evaluated with the BIS scale, 
and, compared to controls, individuals with PHB showed a different pattern of reaction times during go trials, known as an indicator of impulsivity, depending on the presence of addiction-related stimuli. In other words, while individuals with $\mathrm{PHB}$ were faster to respond on go trials with neutral backgrounds, they were slower on trials with sexual backgrounds. This finding is consistent with the fact that individuals with high sexual excitability are thought to exhibit an attentional bias toward sexually arousing stimuli (Macapagal, Janssen, Fridberg, Finn, \& Heiman, 2011), and could indicate that individuals with PHB fail to maintain their response strategy because their attention is occupied by the sexual cues.

The fMRI data collected in this study suggest that the deficit in response inhibition observed in the PHB group is related to altered functions in several brain regions. According to our main effect analysis of trial type, the precentral and postcentral gyrus, which represent the primary sensory and primary motor area, were stronger activated during go than during no-go trials. In addition, the activation in the IFG was greater during no-go than during go trials, which is consistent with previous studies showing that the IFG plays an essential role in successful response inhibition (Aron, Monsell, Sahakian, \& Robbins, 2004; Menon, Adleman, White, Glover, \& Reiss, 2001; Rubia et al., 2001). According to our analysis of main effects of group, the supplementary motor area, IFG, and inferior parietal lobule, which are known to be necessary areas for successful response inhibition, were less activated in individuals with PHB than in the control group, which is consistent with task performance in the current study (Aron, Monsell, Sahakian, \& Robbins, 2004; Duann, Ide, Luo, \& Li, 2009; Simmonds, Pekar, \& Mostofsky, 2008; Swick, Ashley, \& Turken, 2011). In contrast, in the group with $\mathrm{PHB}$, the brain areas known to be related to sensory, motor, and emotional salience as well as to stimulus perception were more active than in the control group. This suggests that task performance was lower in the PHB group than in the control group because of a deficit in response inhibition or low concentration on the task caused by an attentional bias toward other stimuli.

Specifically, these behavioral characteristics of the PHB group may be associated with altered activations in the right fusiform gyrus and right IFG and decreased functional connectivity between the right IFG and the preSMA. We found greater activation in the fusiform gyrus in the PHB group compared to our healthy controls. The fusiform gyrus was much stronger activated when sexual backgrounds were presented than when neutral backgrounds were shown, in all participants. Moreover, this activation in the fusiform gyrus in response to sexual stimuli was enhanced in the PHB group. This activation of the fusiform gyrus might be related to increased attention to targets that are perceived as sexual cues. Previous neuroimaging studies have suggested that face recognition activates the fusiform gyrus, and that the degree of activation in this area appears to rely on the degree of attention toward faces (Gobbini \& Haxby, 2007; O'Craven, Downing, \& Kanwisher, et al., 1999). In the current study, the fusiform gyrus activation could reflect the greater attention that was occupied by sexual cues in subjects with $\mathrm{PHB}$, which then resulted in the higher number of errors during the tasks, compared to the control group.

The activation pattern of the IFG in the current study also supports a deficit in response inhibition and an attentional bias toward sexual cues in the PHB group. In the go/ no-go tasks, our brain imaging results showed no group differences in brain activation during the go trials, but a significant group difference in the activation of the right IFG during the no-go trials, regardless of backgrounds. This difference was particularly enhanced during no-go trials presented on sexual backgrounds. These results are in line with previous neuroimaging studies on response inhibition (Aron, Behrens, Smith, Frank, \& Poldrack, 2007, Aron, Monsell, Sahakian, \& Robbins, 2004; Hampshire, Chamberlain, Monti, Duncan, \& Owen, 2010; Menon, Adleman, White, Glover, \& Reiss, 2001; Rubia et al., 2001), which proposed that the right IFG plays a key role in response inhibition. Hampshire, Chamberlain, Monti, Duncan, \& Owen, 2010 have suggested that the right IFG is responsible for attention shifting, which is known to be an important factor in inhibition. Attention shifting is the process of switching one's attention from one object to another (Monsell, 2003). In other words, the right IFG plays a role in inhibiting attention to one object in order to switch attention to another object. In our study, there was a significant difference in the error rate between sexual and neutral backgrounds during the no-go trials. IFG activity was also higher on trials with sexual backgrounds compared to those with neutral backgrounds. The no-go trials with sexual cues might require additional response inhibition compared to those with neutral cues. Further, they may also require the individual to control their switching of attention towards hedonic information. This may explain why the right IFG could be more activated in conditions where a sexual background is presented than when a neutral background is presented.

Our functional connectivity results support the presence of a structural/functional action control network constituted by the preSMA and right IFG. Previous fMRI studies have shown that both regions are activated during stopping and preparing to stop (Chikazoe et al., 2009; Jahfari, Stinear, Claffey, Verbruggen, \& Aron, 2010; Zandbelt \& Vink, 2010). Other work investigated structural connections within this network and demonstrated that white matter tracts directly connect the right IFG to both the preSMA and the subthalamic nucleus, providing a putative circuit for their interaction during motor control (Aron et al., 2007). Functional connectivity between the right IFG and preSMA has been shown to increase during successful stopping. Results from the Granger causality analysis of the stop signal task suggest that the right IFG influences motor response through its interaction with the preSMA (Duann, Ide, Luo, $\& \mathrm{Li}, 2009)$. These results support the additional possibility that one or both regions are involved in preparing the stopping network prior to the stopping action, so that inhibitory control can be triggered when the stop signal is 
detected (Swann et al., 2012). Similarly, our research found strong between-region coherence during successful response inhibition. This coherence collapsed when the individuals with PHB failed to control behavioral inhibition during exposure to sexual backgrounds. We demonstrate an alteration in inhibitory networks in individuals with $\mathrm{PHB}$, similar to previous studies investigating the connectivity between the right IFG and right preSMA during response inhibition (Duann, Ide, Luo, \& Li, 2009). In summary, our findings suggest that individuals with $\mathrm{PHB}$ have difficulties in inhibiting their responses and that these behavioral characteristics might be associated with the decreased activation of the right IFG and the reduced functional connectivity between the right IFG and preSMA.

The present study has however several limitations. First, the study involved only heterosexual male participants. Future studies should examine female subjects, given that there may be sex-related differences in neural responses to visual sexual stimuli (Hamann, Herman, Nolan, \& Wallen, 2004). Second, individuals with PHB were not diagnosed with any other psychiatric disorders, had no history of criminal behavior, and did not use psychotropic medications. We used these factors as exclusion criteria in order to understand the sole effect of hypersexuality on response inhibition. For instance, there is substantial evidence supporting the relationship between response inhibition and criminal behavior (Chen, Tien, Juan, Tzeng, \& Hung, 2005) or mental disability (Hester \& Garavan, 2004; Kaufman, Ross, Stein, \& Garavan, 2003). Further, the pharmacological effects of psychotropic medications on brain vascular response have also been characterized (Anand et al., 2005). As theses exclusion criteria might limit the generalizability of our results to a broader population, the findings of this study must be interpreted with caution. Another potential limitation in this study was the lack of a control group consisting of adults with attention deficit hyperactivity disorder (ADHD), which has been reported as a common co-occurring diagnosis in approximately $25 \%$ of hypersexual men (Reid, Davtian, Lenartowicz, Torrevillas, \& Fong, 2013). Given that response inhibition has been commonly observed in the ADHD population, it is plausible that our findings may be attributable to ADHD rather than hypersexuality per se.

Despite these limitations, this study makes important contributions to the existing literature and has significant implications for future research. First, our data were obtained from objective assessment tools (go/no-go task) and did not rely on self-reporting methods, which are conventional in studies of individuals with PHB. Second, our findings support difficulties in response inhibition among hypersexual individuals when distracted by sexual stimuli. Such deficits have been observed in many psychiatric populations, including those with substance-related disorders, eating, mood, and anxiety disorders, and behavioral addictions such as gambling disorder. Third, the findings of this study may have therapeutic implications for clinicians working with patients with hypersexual characteristics. For instance, interventions such as neurofeedback or pharmacological treatments that strengthen the cortical regions of the brain potentially implicated in hypersexuality may prove successful in attenuating the symptoms of $\mathrm{PHB}$ in this population.

Funding sources: This work was supported by the Ministry of Education of the Republic of Korea and the National Research Foundation of Korea (NRF-2018S1A5A8029877).

Author contributions: JWS made substantial contributions to conception and design, acquisition of the data, and analysis and interpretation of the data. JWS and JHS were involved in drafting the manuscript and revising it critically for important intellectual content. JWS and JHS agree to be accountable for all aspects of the work in ensuring that questions related to the accuracy or integrity of any part of the work are appropriately investigated and resolved. All authors gave final approval of the version to be published.

Conflict of interest: The research presented here was conducted in the absence of any commercial or financial relationships that could be construed as potential conflicts of interest.

\section{APPENDIX A. SUPPLEMENTARY DATA}

Supplementary data to this article can be found online at https://doi.org/10.1556/2006.2020.00003.

\section{REFERENCES}

Anand, A., Li, Y., Wang, Y., Wu, J., Gao, S., \& Bukhari, L., et al. (2005). Antidepressant effect on connectivity of the moodregulating circuit: An FMRI study. Neuropsychopharmacology, 30(7), 1334-1344. https://doi.org/10.1038/sj.npp.1300725.

Antons, S., \& Brand, M. (2018). Trait and state impulsivity in males with tendency towards Internet-pornography-use disorder. Addictive Behaviors, 79, 171-177. https://doi.org/10.1016/j. addbeh.2017.12.029.

Aron, A. R., Behrens, T. E., Smith, S., Frank, M. J., \& Poldrack, R. A. (2007), The neural basis of inhibition in cognitive control. The Neuroscientist, 13(2), 214-228. https://doi.org/10.1177/ 1073858407299288.

Aron, A. R. (2011). From reactive to proactive and selective control: Developing a richer model for stopping inappropriate responses. Biological Psychiatry, 69(12), e55-e68. https://doi.org/ 10.1016/j.biopsych.2010.07.024.

Aron, A. R., Behrens, T. E., Smith, S., et al. (2007). Triangulating a cognitive control network using diffusion-weighted magnetic resonance imaging (MRI) and functional MRI. Journal of Neuroscience, 27(14), 3743-3752. https://doi.org/10.1523/ JNEUROSCI.0519-07.2007.

Aron, A. R., Monsell, S., Sahakian, B. J., \& Robbins, T. W. (2004). A componential analysis of task switching deficits associated with lesions of left and right frontal cortex. Brain, 127(7), 15611573. https://doi.org/10.1093/brain/awh169. 
Beck, A. T., Steer, R. A., \& Brown, G. K. (1996). Manual for the Beck depression inventory-II. San Antonio, TX: Psychological Corporation.

Carnes, P. (2001). Out of the shadows: Understanding sexual addiction (3rd ed.). Hazelden Publishing.

Carnes, P., Green, B., \& Carnes, S. (2010). The same yet different: Refocusing the Sexual Addiction Screening Test (SAST) to reflect orientation and gender. Sexual Addiction and Compulsivity, 17(1), 7-30. https://doi.org/10.1080/10720161003604087.

Chen, C. Y., Tien, Y. M., Juan, C. H., Tzeng, O. J., \& Hung, D. L. (2005). Neural correlates of impulsive-violent behavior: An event-related potential study. NeuroReport, 16(11), 1213-1216.

Chikazoe, J. (2010). Localizing performance of go/no-go tasks to prefrontal cortical subregions. Current Opinion in Psychiatry, 23(3), 267-272. https://doi.org/10.1097/YCO.0b013e3283387a9f.

Chikazoe, J., Jimura, K., Hirose, S., Yamashita, K. I., Miyashita, Y., \& Konishi, S. (2009). Preparation to inhibit a response complements response inhibition during performance of a stop-signal task. Journal of Neuroscience, 29(50), 15870-15877. https://doi. org/10.1523/JNEUROSCI.3645-09.2009.

Cox, W. M., Hogan, L. M., Kristian, M. R., \& Race, J. H. (2002). Alcohol attentional bias as a predictor of alcohol abusers' treatment outcome. Drug and Alcohol Dependence, 68(3), 237243. https://doi.org/10.1016/S0376-8716(02)00219-3.

Dawe, S., Gullo, M. J., \& Loxton, N. J. (2004). Reward drive and rash impulsiveness as dimensions of impulsivity: Implications for substance misuse. Addictive Behaviors, 29(7), 1389-1405. https://doi.org/10.1016/j.addbeh.2004.06.004.

Dillon, D. G., \& Pizzagalli, D. A. (2007). Inhibition of action, thought, and emotion: A selective neurobiological review. Applied and Preventive Psychology, 12(3), 99-114. https://doi. org/10.1016/j.appsy.2007.09.004.

Ding, W. N., Sun, J. H., Sun, Y. W., Chen, X., Zhou, Y., \& Zhuang, Z. G. (2014). Trait impulsivity and impaired prefrontal impulse inhibition function in adolescents with internet gaming addiction revealed by a Go/No-Go fMRI study. Behavioral and Brain Functions, 10(1), 20. https://doi.org/10.1186/1744-9081-10-20.

Dong, G., Devito, E. E., Du, X., \& Cui, Z. (2012). Impaired inhibitory control in 'internet addiction disorder': A functional magnetic resonance imaging study. Psychiatry Research: Neuroimaging, 203(2-3), 153-158. https://doi.org/10.1016/j.pscychresns.2012.02. 001.

Duann, J. R., Ide, J. S., Luo, X., \& Li, C. S. R. (2009). Functional connectivity delineates distinct roles of the inferior frontal cortex and presupplementary motor area in stop signal inhibition. Journal of Neuroscience, 29(32), 10171-10179. https:// doi.org/10.1523/JNEUROSCI.1300-09.2009.

Field, M., \& Cox, W. M. (2008). Attentional bias in addictive behaviors: A review of its development, causes, and consequences. Drug and Alcohol Dependence, 97(1-2), 1-20. https://doi.org/ 10.1016/j.drugalcdep.2008.03.030.

Ford, A., McGregor, K. M., Case, K, Crosson, B., \& White, K. D. (2010). Structural connectivity of Broca's area and medial frontal cortex. NeuroImage, 52(4), 1230-1237. https://doi.org/10.1016/j. neuroimage.2010.05.018.

Forstmann, B. U., Anwander, A., Schäfer, A., Neumann, J., Brown, S., \& Wagenmakers, E. J. (2010). Cortico-striatal connections predict control over speed and accuracy in perceptual decision making. Proceedings of the National Academy of Sciences USA, 107(36), 15916-15920. https://doi.org/10.1073/pnas. 1004932107.

Franken, I. H. (2003). Drug craving and addiction: Integrating psychological and neuropsychopharmacological approaches. Progress in Neuropsychopharmacology and Biological Psychiatry, 27(4), 563-579. https://doi.org/10.1016/S0278-5846(03)00081-2.

Gobbini, M. I., \& Haxby, J. V. (2007). Neural systems for recognition of familiar faces. Neuropsychologia, 45, 32-41. https:// doi.org/10.1016/j.neuropsychologia.2006.04.015.

Hamann, S., Herman, R. A., Nolan, C. L., \& Wallen, K. (2004). Men and women differ in amygdala response to visual sexual stimuli. Nature Neuroscience, 7(4), 411-416. https://doi.org/10.1038/ nn1208.

Hampshire, A., Chamberlain, S. R., Monti, M. M., Duncan, J., \& Owen, A. M. (2010). The role of the right inferior frontal gyrus: Inhibition and attentional control. NeuroImage, 50(3), 13131319. https://doi.org/10.1016/j.neuroimage.2009.12.109.

Harnishfeger, K. K. (1995). The development of cognitive inhibition. In: F. N. Dempster, \& C. J. Brainerd (Eds.) Interference and inhibition in cognition. (pp. 175-204). Elsevier Inc. https:// doi.org/10.1016/B978-012208930-5/50007-6.

Hester, R., \& Garavan, H. (2004). Executive dysfunction in cocaine addiction: Evidence for discordant frontal, cingulate, and cerebellar activity. Journal of Neuroscience, 24(49), 11017-11022. https://doi.org/10.1523/JNEUROSCI.3321-04.2004.

Jahfari, S., Stinear, C. M., Claffey, M., Verbruggen, F., \& Aron, A. R. (2010). Responding with restraint: What are the neurocognitive mechanisms? Journal of Cognitive Neuroscience, 22(7), 14791492. https://doi.org/10.1162/jocn.2009.21307.

Kafka, M. P. (2010). Hypersexual disorder: A proposed diagnosis for DSM-V. Archives of Sexual Behavior, 39(2), 377-400. https://doi.org/10.1007/s10508-009-9574-7.

Kaufman, J. N., Ross, T. J., Stein, E. A., \& Garavan, H. (2003). Cingulate hypoactivity in cocaine users during a GO-NOGO task as revealed by event-related functional magnetic resonance imaging. Journal of Neuroscience, 23(21), 7839-7843.

Le Marquand, D. G., Pihl, R. O., Young, S. N., Tremblay, R. E., Séguin, J. R., Palmour, R. M., \& Benkelfat, C. (1998). Tryptophan depletion, executive functions, and disinhibition in aggressive, adolescent males. Neuropsychopharmacology, 19(4), 333-341. https://doi.org/10.1016/S0893-133X(98)00029-3.

Levy, B. J., \& Wagner, A. D. (2011). Cognitive control and right ventrolateral prefrontal cortex: Reflexive reorienting, motor inhibition, and action updating. Annals of the New York Academy of Sciences, 1224, 40-62. https://doi.org/10.1111/j. 1749-6632.2011.05958.x.

Liu, G. C., Yen, J. Y., Chen, C. Y., Yen, C. F., Chen, C. S., \& Lin, W. C. (2014). Brain activation for response inhibition under gaming cue distraction in internet gaming disorder. The Kaohsiung Journal of Medical Sciences, 30(1), 43-51. https:// doi.org/10.1016/j.kjms.2013.08.005.

Luijten, M., Littel, M., \& Franken, I. H. (2011). Deficits in inhibitory control in smokers during a Go/NoGo task: An investigation using event-related brain potentials. Public Library of Science One, 6(4), e18898. https://doi.org/10.1371/journal.pone.0018898.

Macapagal, K. R., Janssen, E., Fridberg, D. J., Finn, P. R., \& Heiman, J. R. (2011). The effects of impulsivity, sexual arousability, and 
abstract intellectual ability on men's and women's go/no-go task performance. Archives of Sexual Behavior, 40(5), 995-1006. https://doi.org/10.1007/s10508-010-9676-2.

Mechelmans, D. J., Irvine, M., Banca, P., Porter, L., Mitchell, S., \& Mole, T. B. (2014). Enhanced attentional bias towards sexually explicit cues in individuals with and without compulsive sexual behaviours. PloS One, 9(8), e105476. https://doi.org/10.1371/ journal.pone.0105476.

Menon, V., Adleman, N. E., White, C. D., Glover, G. H., \& Reiss, A. L. (2001). Error-related brain activation during a Go/NoGo response inhibition task. Human Brain Mapping, 12(3), 131143. https://doi.org/10.1002/1097-0193(200103)12:3<131:: AID-HBM1010>3.0.CO;2-C.

Meule, A., Vögele, C., \& Kübler, A. (2011). Psychometrische Evaluation der deutschen Barratt Impulsiveness Scale - Kurzversion (BIS-15). Diagnostica, 57(3), 126-133. http://dx.doi.org/ 10.1026/0012-1924/a000042.

Miner, M. H., Raymond, N., Mueller, B. A., Lloyd, M., \& Lim, K. O., (2009). Preliminary investigation of the impulsive and neuroanatomical characteristics of compulsive sexual behavior. Psychiatry Research, 174(2), 146-151. https://doi.org/10.1016/j. pscychresns.2009.04.008.

Monsell, S. (2003). Task switching. Trends in Cognitive Sciences, 7(3), 134-140. https://doi.org/10.1016/S1364-6613(03)00028-7.

Nachev, P., Kennard, C., \& Husain, M. (2008). Functional role of the supplementary and pre-supplementary motor areas. Nature Reviews Neuroscience, 9(11), 856-869. https://doi.org/10.1038/nrn2478.

Noël, X., Van Der Linden, M., D’Acremont, M., Bechara, A., Dan, B., \& Hanak, C. (2007). Alcohol cues increase cognitive impulsivity in individuals with alcoholism. Psychopharmacology, 192(2), 291-298. https://doi.org/10.1007/s00213-006-0695-6.

O'Craven, K., Downing, P., \& Kanwisher, N. (1999). fMRI evidence for objects as the units of attentional selection. Nature, 401, 584-587. https://doi.org/10.1038/44134.

Odlaug, B. L., Chamberlain, S. R., Kim, S. W., Schreiber, L. R. N., \& Grant, J. E. (2011). A neurocognitive comparison of cognitive flexibility and response inhibition in gamblers with varying degrees of clinical severity. Psychological Medicine, 41, 21112119. https://doi.org/10.1017/S0033291711000316.

Patton, J. H., Stanford, M. S., \& Barratt, E. S. (1995) Factor structure of the Barratt impulsiveness scale. Journal of Clinical Psychology, 51, 768-774. https://doi.org/10.1002/1097-4679(199511)51: 6<768::AID-JCLP2270510607>3.0.CO;2-1.

Reid, R. C., Davtian, M., Lenartowicz, A., Torrevillas, R. M., \& Fong, T. W. (2013). Perspectives on the assessment and treatment of adult ADHD in hypersexual men. Neuropsychiatry, 3(3): 295-308.

Reid, R. C., Garos, S., \& Carpenter, B. N. (2011a). Reliability, validity, and psychometric development of the hypersexual behavior inventory in an outpatient sample of men. Sexual Addiction and Compulsivity, 18(1), 30-51. https://doi.org/10. 1080/10720162.2011.555709.

Reid, R. C., Garos, S., Carpenter, B. N., \& Coleman, E. (2011b). A surprising finding related to executive control in a patient sample of hypersexual men. The Journal of Sexual Medicine, 8(8), 2227-2236. https://doi.org/10.1111/j.1743-6109.2011. 02314.x.

Reid, R. C., Karim, R., McCrory, E., \& Carpenter, B. N. (2010). Selfreported differences on measures of executive function and hypersexual behavior in a patient and community sample of men. International Journal of Neuroscience, 120(2), 120-127. https://doi.org/10.3109/00207450903165577.

Rubia, K., Russell, T., Overmeyer, S., Brammer, M. J., Bullmore, E. T., \& Sharma, T. (2001). Mapping motor inhibition: Conjunctive brain activations across different versions of go/ no-go and stop tasks. NeuroImage, 13(2), 250-261. https://doi. org/10.1006/nimg.2000.0685.

Seok, J. W., \& Sohn, J. H. (2018). Altered prefrontal and inferior parietal activity during a stroop task in individuals with problematic hypersexual behavior. Frontiers in Psychiatry, 9, 460. https://doi.org/10.3389/fpsyt.2018.00460.

Simmonds, D. J., Pekar, J. J., \& Mostofsky, S. H. (2008). Metaanalysis of Go/No-go tasks demonstrating that fMRI activation associated with response inhibition is task-dependent. Neuropsychologia, 46(1), 224-232. https://doi.org/10. 1016/j.neuropsychologia.2007.07.015.

Swann, N. C., Cai, W., Conner, C. R., Pieters, T. A., Claffey, M. P., \& George, J. S. (2012). Roles for the pre-supplementary motor area and the right inferior frontal gyrus in stopping action: Electrophysiological responses and functional and structural connectivity. NeuroImage, 59(3), 2860-2870. https://doi.org/10.1016/j.neuroimage.2011.09.049.

Swick, D., Ashley, V., \& Turken, U. (2011). Are the neural correlates of stopping and not going identical? Quantitative meta-analysis of two response inhibition tasks. NeuroImage, 56(3), 1655-1665. https://doi.org/10.1016/j.neuroimage.2011.02.070.

Telzer, E. H., Fuligni, A. J., Lieberman, M. D., \& Galván, A. (2013). Meaningful family relationships: Neurocognitive buffers of adolescent risk taking. Journal of Cognitive Neuroscience, 25(3), 374-387. https://doi.org/10.1162/jocn_a_00331.

Van Holst, Van Holstein, M., Van Den Brink, W., Veltman, A. E., \& Goudriaan, C. D. (2012). Response inhibition during cue reactivity in problem gamblers: An fMRI study. Plos One, 7(3), e30909. https://doi.org/10.1371/journal.pone. 0030909 .

Zandbelt, B. B., \& Vink, M. (2010). On the role of the striatum in response inhibition. Public Library of Science One, 5(11), e13848e13848. https://doi.org/10.1371/journal.pone.0013848. 